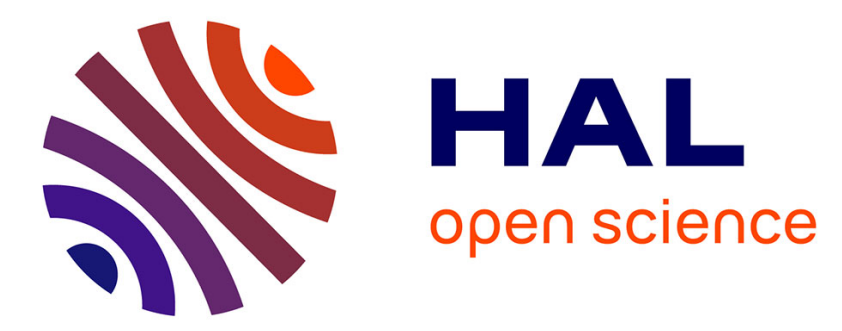

\title{
Application of the virtual work principle to compute magnetic forces with a volume integral method
}

Anthony Carpentier, Nicolas Galopin, Olivier Chadebec, Gérard Meunier, Christophe Guérin

\section{- To cite this version:}

Anthony Carpentier, Nicolas Galopin, Olivier Chadebec, Gérard Meunier, Christophe Guérin. Application of the virtual work principle to compute magnetic forces with a volume integral method. International Journal of Numerical Modelling: Electronics Networks, Devices and Fields, 2013, 27 (3), pp.418-432. 10.1002/jnm.1957 . hal-00996626

\section{HAL Id: hal-00996626 https://hal.science/hal-00996626}

Submitted on 2 Feb 2021

HAL is a multi-disciplinary open access archive for the deposit and dissemination of scientific research documents, whether they are published or not. The documents may come from teaching and research institutions in France or abroad, or from public or private research centers.
L'archive ouverte pluridisciplinaire HAL, est destinée au dépôt et à la diffusion de documents scientifiques de niveau recherche, publiés ou non, émanant des établissements d'enseignement et de recherche français ou étrangers, des laboratoires publics ou privés. 


\title{
Application of the virtual work principle to compute magnetic forces with a volume integral method
}

\author{
Anthony Carpentier ${ }^{1}$, Nicolas Galopin ${ }^{1, *, \dagger}{ }^{\dagger}$, Olivier Chadebec ${ }^{1,2}$, Gérard Meunier ${ }^{1}$ and \\ Christophe Guérin ${ }^{3}$ \\ ${ }^{1}$ Grenoble Electrical Engineering Laboratory, UMR CNRS 5269, Grenoble INP, UJF, University of Grenoble, France \\ ${ }^{2}$ GRUCAD/EEL/CTC/UFSC, Florianópolis, Brazil \\ ${ }^{3}$ CEDRAT S.A., 15 Chemin de Malacher, 38240 Meylan, France
}

\begin{abstract}
SUMMARY
Magnetic forces are computed in the scope of magnetostatic problems solved by a volume integral method. This method uses the range interactions between magnetizable elements and is particularly well suited to compute the fields without meshing the air domain. This paper proposes an adaptation of the virtual work principle to the framework of the volume integral method in order to compute the magnetic forces. First, its application for electromagnetic devices in the nonlinear case allows to compute the global magnetic force. Second, its local application in the linear case provides a magnetic force density. 3D numerical examples are given, and the results are compared with the analytical solution and the finite element method. Copyright (C 2013 John Wiley \& Sons, Ltd.
\end{abstract}

KEY WORDS: magnetic force; integral method; virtual works; magnetostatics

\section{INTRODUCTION}

In a design process, the modeling of electromechanical devices commonly involves precisely determining the forces acting on ferromagnetic materials. For a rigid motion, only the knowledge of the global force is necessary, however, for a device with deformable material, it is essential to know the local force distribution. A classical method to numerically solve such problems is the finite element one (FEM), but for devices with a huge volume of free space compared with the active structure or with a high size ratio between the geometric objects, problems of accuracy and convergence to the solution can be present [1]. In such case, integral methods are attractive alternatives [2].

Integral formulations of the magnetostatic problem are particularly advantageous for the numerical solution of open-boundary problems, which include ferromagnetic materials, because only the active regions containing these materials need to be discretized. This is particularly useful for problems with a lot of air compared with active regions, such as Mag-MEMS devices [3]. In this case, integral methods lead to high accuracies for field computations [4]. A volume integral method (VIM) using the magnetic scalar potential is used.

The computation of the magnetic force can be conducted by different methods [5]: Maxwell's tensor, virtual works, equivalent currents or charges, eggshell method [6], and so on. These methods provide global magnetic forces that converge to the same value in spite of different densities, some of which are improbable [7]. The virtual work method is more general [8] and provides good results in the framework of the finite element method [9]. Few works deal with magnetic forces in the framework of

*Correspondence to: Nicolas Galopin, G2elab, Ense3, 11 rue des Mathématiques, BP 46, 38402 Saint-Martin d'Hères Cedex, France.

${ }^{\dagger}$ E-mail: nicolas.galopin@g2elab.grenoble-inp.fr 
the integral formulations, and when this is done, they are restricted to the computation of global forces [10]. So, an adaptation of the virtual work principle to the VIM is proposed.

This paper presents in the first part a magnetostatic formulation in the framework of VIM. The second part is dedicated to the adaptation of the virtual work principle to this formulation. In the linear case, a local force calculation method based on local application of virtual work principle is also proposed. The last one presents the results obtained with two applications.

\section{VOLUME INTEGRAL METHOD}

Let us consider the following magnetostatic problem (Figure 1). A three-dimensional simply connected region $\Omega_{f}$ is filled with isotropic ferromagnetic material characterized by the known magnetic susceptibility $\chi$, such as

$$
\mathbf{M}=\chi(\|\mathbf{H}\|) \mathbf{H},
$$

where $\mathbf{M}$ is the magnetization and $\mathbf{H}$ the magnetic field. Primary sources of magnetic field in which currents flow are associated to the region $\Omega_{j}$. Both regions, $\Omega_{f}$ and $\Omega_{j}$, are in free space $\Omega_{0}$ so that these regions do not overlap, $\Omega=\Omega_{f} \cup \Omega_{j} \cup \Omega_{0}$. We note $\Gamma$ the boundary between the ferromagnetic material $\Omega_{f}$ and the free space $\Omega_{0}$. As the simply connected region $\Omega_{f}$ contains no current sources and according to the Maxwell's equations, the magnetic field $\mathbf{H}$ derives from a magnetic scalar potential $\Phi$ in the region $\Omega_{f}$ :

$$
\mathbf{H}=-\nabla \Phi
$$

The source magnetic field $\mathbf{H}_{0}$ also derives from a magnetic scalar potential $\Phi_{0}$ :

$$
\mathbf{H}_{0}=-\nabla \Phi_{0}
$$

The following volume integral equation using the total scalar potential $\Phi[11]$ is considered :

$$
\Phi(\mathbf{r})-\frac{1}{4 \pi} \int_{\Omega_{f}} \frac{\mathbf{M}\left(\mathbf{r}^{\prime}\right) \cdot\left(\mathbf{r}-\mathbf{r}^{\prime}\right)}{\left|\mathbf{r}-\mathbf{r}^{\prime}\right|^{3}} \mathrm{~d} \Omega^{\prime}=\Phi_{0}(\mathbf{r}), \forall \mathbf{r} \in \Omega_{f},
$$

where $\mathbf{r}$ and $\mathbf{r}^{\prime}$ are respectively the coordinates of the computation and integration points. For notational convenience, the dependency of variables and functions on computation and integration points is often omitted.

In accordance with the advantage of the VIM, only the domain $\Omega_{f}$ is meshed. A tetrahedral mesh is used, and the potential $\Phi$ is approximated by first-order nodal shape functions:

$$
\Phi=\sum_{i} \Phi_{i} \alpha_{i}
$$

where $\Phi_{i}$ and $\alpha_{i}$ are respectively the degree of freedom (DOF) of the potential $\Phi$ and the first-order nodal shape function associated with the mesh node $i$.

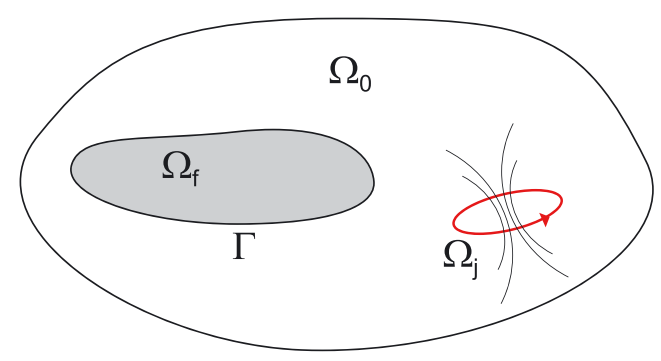

Figure 1. Description of the magnetostatic problem. 
In the linear case, Equation (4) is solved using a collocation method at mesh nodes [12]. It leads to the system of algebraic equations:

$$
([I]+[A(\chi)]) \Phi=\boldsymbol{\Phi}_{0},
$$

with

$$
\begin{gathered}
(I)_{i j}=\delta_{i j}, \\
(A(\chi))_{i j}=\frac{1}{4 \pi} \int_{\Omega_{f}} \chi\left(\mathbf{r}^{\prime}\right) \frac{\nabla \alpha_{j}\left(\mathbf{r}^{\prime}\right) \cdot\left(\mathbf{r}_{i}-\mathbf{r}^{\prime}\right)}{\left|\mathbf{r}_{i}-\mathbf{r}^{\prime}\right|^{3}} \mathrm{~d} \Omega^{\prime},
\end{gathered}
$$

where $\mathbf{r}_{i}$ is the coordinates of the mesh node $i$, and $\boldsymbol{\Phi}$ and $\boldsymbol{\Phi}_{0}$ are the vectors of DOFs associated respectively to the potentials $\Phi$ and $\Phi_{0}$. In the nonlinear case, a fixed point scheme is used on Equation (4) [13].

If the domain $\Omega_{f}$ is not simply connected, a cut is used to split it into simply connected domains. A drawback of the VIM is the obtention of a full interaction matrix (8) making the resolution of problems that require fine meshes difficult. That is why compression techniques could be applied to avoid this limitation [14,15]. Moreover the computation of the interaction matrix (8) requires precautions for the evaluation of the integral $I_{1}$ on each element [13]:

$$
I_{1}(\mathbf{r})=\int_{\Omega_{e}} \mathbf{S} \cdot \frac{\left(\mathbf{r}-\mathbf{r}^{\prime}\right)}{\left|\mathbf{r}-\mathbf{r}^{\prime}\right|^{3}} \mathrm{~d} \Omega^{\prime}
$$

where $\Omega_{e}$ is the domain of the element $e$ and $\mathbf{S}$ is a uniform vector corresponding to $\chi \nabla \alpha_{i}$, which are uniform on each mesh element according to the first-order discretization (5) and the tetrahedral mesh. When the computation point $\mathbf{r}$ is closed to the integration point $\mathbf{r}^{\prime}$, an analytical formula [16] is considered to evaluate (9); otherwise, a numerical integration scheme with Gauss points is used.

In order to compute the source potential $\Phi_{0}$, the minimization problem associated to the equation (3) is considered:

$$
\min _{\Phi_{0}}\left[\left(\mathbf{H}_{0}+\nabla \Phi_{0}\right)^{2}\right]
$$

where $\mathbf{H}_{0}$ can be computed analytically [17]. In the domain $\Omega_{f}$, the potential $\Phi_{0}$ is approximated with first-order nodal shape functions similar to Equation (5). A FEM is used on the weak formulation associated to the problem (10). It leads to the resolution of a system of algebraic equations:

$$
[D] \Phi_{0}=\mathbf{C}
$$

with

$$
\begin{aligned}
& (D)_{i j}=\int_{\Omega_{f}} \nabla \alpha_{i} \cdot \nabla \alpha_{j} \mathrm{~d} \Omega, \\
& (C)_{i}=-\int_{\Omega_{f}} \nabla \alpha_{i} \cdot \mathbf{H}_{0} \mathrm{~d} \Omega .
\end{aligned}
$$

\section{VIRTUAL WORK PRINCIPLE}

The magnetostatic problem is solved using the previous VIM. The vectors of DOFs $\boldsymbol{\Phi}$ and $\boldsymbol{\Phi}_{0}$ are then known and verify respectively relations (6) and (11). The susceptibility is computed using the magnetic behavior law (1). 


\subsection{Application on a magneto-mechanical system}

Let us consider that the source field is created by a coil without resistance. According to the virtual work principle, the application of energy conservation law to a magneto-mechanical system without losses [18] gives

$$
\Psi \mathrm{d} I=-\mathrm{d} \omega_{\mathrm{mag}}^{\mathrm{co}}+\mathbf{F} \cdot \mathrm{d} \mathbf{u}
$$

where $I$ is the electric current in the coil, $\Psi$ the magnetic flux through the coil section, $\omega_{\mathrm{mag}}^{\mathrm{co}}$ the magnetic co-energy, $\mathbf{F}$ the magnetic force, and $\mathbf{u}$ a virtual displacement of the system.

The magnetic co-energy is defined by

$$
\omega_{\mathrm{mag}}^{\mathrm{co}}=\int_{\Omega}\left(\int_{0}^{\mathbf{H}} \mathbf{B} \cdot \mathrm{d} \mathbf{H}\right) \mathrm{d} \Omega
$$

By using the continuity of the normal component of the magnetic induction through the interface $\Gamma$ and vector identities, the expression of the magnetic co-energy (15) can be written as [19]

$$
\omega_{\mathrm{mag}}^{\mathrm{co}}=\omega_{\text {ferro }}^{\mathrm{co}}+\omega_{0},
$$

where

$$
\begin{gathered}
\omega_{\text {ferro }}^{\mathrm{co}}=\frac{1}{2} \int_{\Omega_{f}}\left(\mathbf{M} \cdot \mathbf{B}_{0}-\mathbf{H} \cdot \mathbf{B}+2 \int_{0}^{\mathbf{H}} \mathbf{B} \cdot \mathrm{d} \mathbf{H}\right) \mathrm{d} \Omega, \\
\omega_{0}=\frac{1}{2} \int_{\Omega} \mathbf{H}_{0} \cdot \mathbf{B}_{0} \mathrm{~d} \Omega .
\end{gathered}
$$

The interest to use this decomposition (16) for the co-energy $\omega_{\mathrm{mag}}^{\mathrm{co}}$ is that the integration domain of the term $\omega_{\text {ferro }}^{\text {co }}$ is only the ferromagnetic material and that the term $\omega_{0}^{\mathrm{co}}$ depends only of the current source.

When the current $I$ is kept constant during the displacement $\mathbf{u}$, the component in the direction $\mathbf{u}$ of the magnetic force, $F_{u}$, can be computed by

$$
F_{u}=\left.\frac{\mathrm{d} \omega_{\mathrm{mag}}^{\mathrm{co}}}{\mathrm{du}}\right|_{\mathbf{I}}
$$

To simplify the notation, the condition of the coil current is not noted. As the energy $\omega_{0}(18)$ is independent of the displacement $\mathbf{u}$, Equation (19) becomes:

$$
F_{u}=\frac{\mathrm{d} \omega_{\text {ferro }}^{\mathrm{co}}}{\mathrm{du}}
$$

Three virtual displacements $\mathbf{u}_{x}, \mathbf{u}_{y}$, and $\mathbf{u}_{z}$ are respectively taken as the three directions of the problem basis $(\mathbf{x}, \mathbf{y}, \mathbf{z})$. The magnetic force can then be expressed:

$$
\mathbf{F}=\frac{\mathrm{d} \omega_{\text {ferro }}^{\mathrm{co}}}{\mathrm{d} \mathbf{u}_{x}} \mathbf{x}+\frac{\mathrm{d} \omega_{\text {ferro }}^{\mathrm{co}}}{\mathrm{d} \mathbf{u}_{y}} \mathbf{y}+\frac{\mathrm{d} \omega_{\text {ferro }}^{\mathrm{co}}}{\mathrm{d} \mathbf{u}_{z}} \mathbf{z} .
$$

\subsection{Global magnetic force computation}

The virtual displacement $\mathbf{u}$ is applied on a piece of the ferromagnetic domain $\Omega_{f}$ provided that the mesh is not distorted. It means that this piece must be surrounded by the free space. The application of 
the virtual work principle (21) allows computing the global magnetic force acting on this piece. The derivative of the co-energy $\omega_{\text {ferro }}^{\text {co }}(17)$ with respect to the displacement $\mathbf{u}$ can be written:

$$
\frac{\mathrm{d} \omega_{\text {ferro }}^{\mathrm{co}}}{\mathrm{d} \mathbf{u}}=\frac{1}{2} \int_{\Omega_{f}}\left(\mathbf{M} \cdot \frac{\mathrm{d} \mathbf{B}_{0}}{\mathrm{~d} \mathbf{u}}+\frac{\mathrm{d} \mathbf{M}}{\mathrm{d} \mathbf{u}} \cdot \mathbf{B}_{0}+\mathbf{B} \cdot \frac{\mathrm{d} \mathbf{H}}{\mathrm{d} \mathbf{u}}-\frac{\mathrm{d} \mathbf{B}}{\mathrm{d} \mathbf{u}} \cdot \mathbf{H}\right) \mathrm{d} \Omega .
$$

The computation of each term of Equation (22) is detailed in the succeeding texts. As the mesh is not distorted, the shape functions are not modified by the displacement:

$$
\frac{\mathrm{d} \alpha_{i}}{\mathrm{du}}=0, \forall i
$$

Computation of $\frac{\mathbf{d B}_{0}}{\mathbf{d u}}$ : The derivation of the source induction field $\mathbf{B}_{0}$ with respect to the displacement u can be written:

$$
\frac{\mathrm{d} \mathbf{B}_{0}(\mathbf{r})}{\mathrm{d} \mathbf{u}}=\mu_{0} \nabla \mathbf{H}_{0}(\mathbf{r}) \frac{\mathrm{d} \mathbf{r}}{\mathrm{d} \mathbf{u}}
$$

The gradient of the magnetic field created by the inductors $\nabla \mathbf{H}_{0}$ can be computed analytically for simple geometries [17]. For more complicate inductor geometries, a finite difference method can be used. The term $\frac{\mathrm{dr}}{\mathrm{du}}$ is equivalent to the direction of the virtual displacement in the moved piece and to the zero vector elsewhere.

Computation of $\frac{\mathbf{d} \mathbf{B}_{0}}{\mathbf{d u}}$ : With Equations (2), (23) and the discretization (5) of the potential $\Phi$, it can be written as

$$
\frac{\mathrm{d} \mathbf{H}}{\mathrm{d} \mathbf{u}}=-\sum_{i} \frac{\mathrm{d} \Phi_{i}}{\mathrm{~d} \mathbf{u}} \nabla \alpha_{i}
$$

Let us note $\frac{\mathrm{d} \Phi}{\mathrm{d} \mathbf{u}}$ the vector of the derivative of the DOFs of the potential $\Phi$ with respect to the displacement $\mathbf{u}$. The derivation of the system of algebraic equations (6) with respect to the displacement u gives

$$
\frac{\mathrm{d} \Phi}{\mathrm{d} \mathbf{u}}+\frac{\mathrm{d}[A(\chi)] \Phi}{\mathrm{du}}=\frac{\mathrm{d} \boldsymbol{\Phi}_{0}}{\mathrm{du}}
$$

$\frac{\mathrm{d} \boldsymbol{\Phi}_{0}}{\mathrm{du}}$ is the vector of the derivative of the DOFs of the potential $\Phi_{0}$ with respect to the displacement $\mathbf{u}$, and its computation is detailed in the next paragraph. The chain rule in the second derivative of Equation (26) leads to

$$
\frac{\mathrm{d}[A(\chi)] \boldsymbol{\Phi}}{\mathrm{du}}=\left.\frac{\partial[A(\chi)] \boldsymbol{\Phi}}{\partial \Phi}\right|_{\mathbf{u}} \frac{\mathrm{d} \boldsymbol{\Phi}}{\mathrm{du}}+\left.\frac{\partial[A(\chi)]}{\partial \mathbf{u}}\right|_{\boldsymbol{\Phi}} \boldsymbol{\Phi}
$$

The matrix $\left[\left.\frac{\partial[A(\chi)] \Phi}{\partial \Phi}\right|_{\mathbf{u}}\right]$ is expressed

$$
\left(\left.\frac{\partial[A(\chi)] \Phi}{\partial \Phi}\right|_{\mathbf{u}}\right)_{i j}=-\int_{\Omega_{f}} \frac{\mathrm{d} \mathbf{M}}{\mathrm{d} \Phi_{j}} \cdot \frac{\left(\mathbf{r}_{i}-\mathbf{r}^{\prime}\right)}{\left|\mathbf{r}_{i}-\mathbf{r}^{\prime}\right|^{3}} \mathrm{~d} \Omega^{\prime}
$$

The derivation of the behavior law (1) with respect to the DOFs of the potential $\Phi$ gives

$$
\frac{\mathrm{d} \mathbf{M}}{\mathrm{d} \Phi_{j}}=\frac{\mathrm{d} \mathbf{M}}{\mathrm{d} \mathbf{H}} \frac{\mathrm{d} \mathbf{H}}{\mathrm{d} \Phi_{j}}=-\frac{\mathrm{d} \mathbf{M}}{\mathrm{d} \mathbf{H}} \nabla \alpha_{j} .
$$


Using relation (29), Equation (28) becomes

$$
\left(\left.\frac{\partial[A(\chi)] \Phi}{\partial \Phi}\right|_{\mathbf{u}}\right)_{i j}=\int_{\Omega_{f}} \frac{\mathrm{d} \mathbf{M}}{\mathrm{d} \mathbf{H}} \nabla \alpha_{j} \cdot \frac{\left(\mathbf{r}_{i}-\mathbf{r}^{\prime}\right)}{\left|\mathbf{r}_{i}-\mathbf{r}^{\prime}\right|^{3}} \mathrm{~d} \Omega^{\prime}
$$

The matrix (30) is equivalent to the matrix (8) where the susceptibility $\chi$ is replaced by the incremental susceptibility $\frac{\mathrm{d} \mathbf{M}}{\mathrm{d} \mathbf{H}}$ defined by

$$
\left(\frac{\mathrm{d} \mathbf{M}}{\mathrm{d} \mathbf{H}}\right)_{i j}=\delta_{i j} \chi(\|\mathbf{H}\|)+\frac{H_{i} H_{j}}{\|\mathbf{H}\|} \frac{\mathrm{d} \chi}{\mathrm{d}\|\mathbf{H}\|}(\|\mathbf{H}\|)
$$

Let $a$ be the integrand of the interaction matrix terms (8), such as

$$
(A)_{i j}=\int_{\Omega_{f}} a\left(\chi \nabla \alpha_{j}, \mathbf{r}_{\mathbf{i}}, \mathbf{r}^{\prime}\right) \mathrm{d} \Omega^{\prime}
$$

with

$$
a\left(\chi \nabla \alpha_{j}, \mathbf{r}_{\mathbf{i}}, \mathbf{r}^{\prime}\right)=\chi \nabla \alpha_{j} \cdot \frac{\left(\mathbf{r}_{\mathbf{i}}-\mathbf{r}^{\prime}\right)}{\left|\mathbf{r}_{\mathbf{i}}-\mathbf{r}^{\prime}\right|^{3}}
$$

As the mesh is not distorted by the virtual displacement $\mathbf{u}$, the derivative of Equation (32) can enter the integral to obtain

$$
\left(\left.\frac{\partial[A(\chi)]}{\partial \mathbf{u}}\right|_{\Phi}\right)_{i j}=\left.\int_{\Omega_{f}} \frac{\partial a\left(\chi \nabla \alpha_{j}, \mathbf{r}_{i}, \mathbf{r}^{\prime}\right)}{\partial \mathbf{u}}\right|_{\Phi} \mathrm{d} \Omega^{\prime}
$$

The function $a$ must be derived with respect to the virtual displacement $\mathbf{u}$ keeping constant the vector of the DOFs. This last condition is equivalent to keep constant the first parameter of the function $\chi \nabla \alpha_{j}$. So, the chain rule is applied to the derivative of Equation (33) and leads to

$$
\left.\frac{\partial a\left(\chi \nabla \alpha_{j}, \mathbf{r}_{i}, \mathbf{r}^{\prime}\right)}{\partial \mathbf{u}}\right|_{\Phi}=\left.\frac{\partial a\left(\chi \nabla \alpha_{j}, \mathbf{r}_{i}, \mathbf{r}^{\prime}\right)}{\partial \mathbf{r}_{i}}\right|_{\Phi, \mathbf{r}^{\prime}} \frac{\mathrm{d} \mathbf{r}_{i}}{\mathrm{~d} \mathbf{u}}+\left.\frac{\partial a\left(\chi \nabla \alpha_{j}, \mathbf{r}_{i}, \mathbf{r}^{\prime}\right)}{\partial \mathbf{r}^{\prime}}\right|_{\Phi, \mathbf{r}_{i}} \frac{\mathrm{d} \mathbf{r}^{\prime}}{\mathrm{d} \mathbf{u}}
$$

The terms $\frac{\mathrm{d} \mathbf{r}_{i}}{\mathrm{~d} \mathbf{u}}$ and $\frac{\mathrm{d} \mathbf{r}^{\prime}}{\mathrm{du}}$ are equivalent to the direction of the virtual displacement in the moved piece and to the zero vector elsewhere.

The computation of term (34) requires the evaluation of the integral $\mathbf{I}_{2}$, such as

$$
\mathbf{I}_{2}(\mathbf{r})=\int_{\Omega_{e}} 3 \frac{\left(\mathbf{S} \cdot\left(\mathbf{r}-\mathbf{r}^{\prime}\right)\right)\left(\mathbf{r}-\mathbf{r}^{\prime}\right)}{\left|\mathbf{r}-\mathbf{r}^{\prime}\right|^{5}}-\frac{\mathbf{S}}{\left|\mathbf{r}-\mathbf{r}^{\prime}\right|^{3}} \mathrm{~d} \Omega^{\prime},
$$

where $\mathbf{S}$ is a uniform vector (9). The integral (36) is computed by a numerical integration scheme with Gauss points because when the terms $\frac{d \mathbf{r}}{\mathrm{du}}$ and $\frac{\mathrm{d} \mathbf{r}^{\prime}}{\mathrm{du}}$ are not zero, the computation point $\mathbf{r}$ is generally distant from the integration point $\mathbf{r}^{\prime}$.

Finally, the vector $\frac{\mathrm{d} \Phi}{\mathrm{d} \mathbf{u}}$ is computed by resolution of the system of algebraic equations:

$$
\left([I]+\left[A\left(\frac{\mathrm{d} \mathbf{M}}{\mathrm{d} \mathbf{H}}\right)\right]\right) \frac{\mathrm{d} \boldsymbol{\Phi}}{\mathrm{du}}=\frac{\mathrm{d} \boldsymbol{\Phi}_{0}}{\mathrm{du}}-\left.\frac{\partial[A(\chi)]}{\partial \mathbf{u}}\right|_{\boldsymbol{\Phi}} \boldsymbol{\Phi} .
$$


Computation of $\frac{\mathrm{d} \boldsymbol{\Phi}_{0}}{\mathrm{du}}$ : Taking into account condition (23), the vector $\frac{\mathrm{d} \boldsymbol{\Phi}_{0}}{\mathrm{du}}$ is the solution of the derivation of the system of algebraic equations (11) with respect to the displacement $\mathbf{u}$ :

$$
[D] \frac{\mathrm{d} \boldsymbol{\Phi}_{0}}{\mathrm{du}}=\frac{\mathrm{d} \mathbf{C}}{\mathrm{du}}
$$

where

$$
\left(\frac{\mathrm{d} \mathbf{C}}{\mathrm{d} \mathbf{u}}\right)_{i}=-\int_{\Omega_{f}} \nabla \alpha_{i} \cdot \frac{\mathrm{d} \mathbf{H}_{0}}{\mathrm{~d} \mathbf{u}} \mathrm{d} \Omega=-\int_{\Omega_{f}} \nabla \alpha_{i}(\mathbf{r}) \cdot \nabla \mathbf{H}_{0}(\mathbf{r}) \frac{\mathrm{d} \mathbf{r}}{\mathrm{d} \mathbf{u}} \mathrm{d} \Omega .
$$

Computation of $\frac{\mathbf{d M}}{\mathbf{d u}}$ and $\frac{\mathbf{d B}}{\mathbf{d u}}$ The chain rule applied to the computation of $\frac{\mathrm{d} \mathbf{M}}{\mathrm{du}}$ and $\frac{\mathrm{d} \mathbf{B}}{\mathrm{du}}$ leads to

$$
\frac{\mathrm{d} \mathbf{M}}{\mathrm{d} \mathbf{u}}=\frac{\mathrm{d} \mathbf{M}}{\mathrm{d} \mathbf{H}} \frac{\mathrm{d} \mathbf{H}}{\mathrm{du}}
$$

and

$$
\frac{\mathrm{d} \mathbf{B}}{\mathrm{du}}=\frac{\mathrm{d} \mathbf{B}}{\mathrm{d} \mathbf{H}} \frac{\mathrm{d} \mathbf{H}}{\mathrm{du}}
$$

where $\frac{d \mathbf{B}}{\mathrm{d} \mathbf{H}}$ is the incremental susceptibility similar to (31).

To conclude this section, the proposed application of the virtual work principle to compute the global magnetic force required the resolution of both systems of algebraic equations (38) and (37). This computation can be conducted efficiently when a Newton-Raphson (NR) method is used to solve the problem (4). Indeed, the matrix $\left([I]+\left[A\left(\frac{\mathrm{d} \mathbf{M}}{\mathrm{d} \mathbf{H}}\right)\right]\right)$ on the left hand side of the system (37) is then equivalent to the Jacobian matrix during the last iteration of the NR method. Moreover, the matrices on the left hand side of both systems (38) and (11) are the same. In this case, the resolution processes could be kept modifying only the right hand members.

\subsection{Local magnetic force computation}

Only the linear case is considered in this section. In order to compute the local magnetic force, the virtual work principle is applied at the mesh node level [9] (Figure 2). The virtual displacement $\mathbf{u}^{n}$ is applied at the mesh node $n$. In this case, the mesh is distorted implying that condition (23) is not verified. Let $\mathbf{F}_{u}^{n}$ be the component of the nodal magnetic force $\mathbf{F}^{n}$ in the direction of the virtual displacement $\mathbf{u}^{n}$. It is obtained by

$$
F_{u}^{n}=\frac{\mathrm{d} \omega_{\text {ferro }}^{\mathrm{co}}}{\mathrm{d} \mathbf{u}^{n}}=\sum_{e \in \Omega_{f}} \frac{\mathrm{d} \omega_{\mathrm{e}}^{\mathrm{co}}}{\mathrm{d} \mathbf{u}^{n}}
$$

where $\omega_{\mathrm{e}}^{\mathrm{co}}$ is the value of the co-energy $\omega_{\mathrm{ferro}}^{\mathrm{co}}$ at the mesh element $e$. According to expression (17) and the assumption of linear case, the co-energy $\omega_{\mathrm{e}}^{\mathrm{co}}$ at each mesh element is then written as
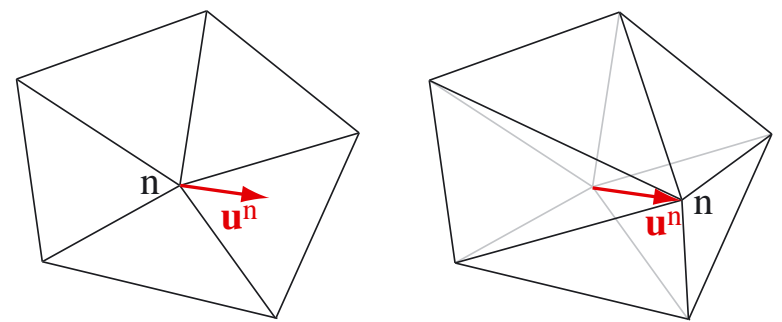

Figure 2. Local application of the virtual work principle (2D mesh example). 


$$
\omega_{\mathrm{e}}^{\mathrm{co}}=\frac{1}{2} \int_{\Omega_{e}} \mathbf{M} \cdot \mathbf{B}_{0} \mathrm{~d} \Omega
$$

The integrals are computed using the change of basis between the physical mesh element and the reference element described in Figure 3. The function $G_{e}$ is the transformation from the reference element of domain $\hat{\Omega}$ to the $e_{t h}$ physical mesh element of domain $\Omega_{e}$, and $J_{e}$ is its Jacobian. Let us note with " "' a function expressed on the basis $(\hat{\mathbf{x}}, \hat{\mathbf{y}}, \hat{\mathbf{z}})$ of the reference element. Because a tetrahedral mesh is used, the gradients of the shape functions are independent of integration points. By using the change of basis, its derivative with respect to the nodal displacement $\mathbf{u}^{n}$ can be computed on an element $e$ by

$$
\frac{\left.\mathrm{d} \nabla \alpha_{i}\right|_{\Omega_{e}}}{\mathrm{~d} \mathbf{u}^{n}}=\frac{\mathrm{d} J_{e}^{-T}}{\mathrm{~d} \mathbf{u}^{n}} \hat{\nabla} \hat{\alpha}_{i}, \forall i
$$

So, Equation (43) is equivalent to

$$
\omega_{\mathrm{e}}^{\mathrm{co}}=\frac{1}{2} \int_{\hat{\Omega}} \mathbf{M} \cdot \mathbf{B}_{0}\left|J_{e}\right| \mathrm{d} \Omega .
$$

Let $F_{u}^{n, e}$ be the contribution of the mesh element $e$ to the component of the nodal magnetic force $F_{u}^{n}$, such as

$$
F_{u}^{n}=\sum_{e} F_{u}^{n, e}
$$

with

$$
F_{u}^{n, e}=\frac{1}{2} \int_{\hat{\Omega}}\left[\left(\mathbf{M} \cdot \frac{\mathrm{d} \mathbf{B}_{0}}{\mathrm{~d} \mathbf{u}^{n}}+\frac{\mathrm{d} \mathbf{M}}{\mathrm{d} \mathbf{u}^{n}} \cdot \mathbf{B}_{0}\right)\left|J_{e}\right|+\left(\mathbf{M} \cdot \mathbf{B}_{0}\right) \frac{\mathrm{d}\left|J_{e}\right|}{\mathrm{d} \mathbf{u}^{n}}\right] \mathrm{d} \Omega .
$$

The computation of each term of equation (47) are detailed below. Those of $\frac{\mathrm{d} J_{e}^{-T}}{\mathrm{~d} \mathbf{u}^{n}}$ and $\frac{\mathrm{d}\left|J_{e}\right|}{\mathrm{d} \mathbf{u}^{n}}$ are obvious.

Computation of $\frac{\mathbf{d B}_{0}}{\mathbf{d u}^{n}}$ : By using the change of basis (Figure 3), the derivative of the source induction field $\mathbf{B}_{0}$ with respect to the nodal displacement $\mathbf{u}^{n}$ can be computed on an element $e$ by

$$
\left.\frac{\mathrm{d} \mathbf{B}_{0}}{\mathrm{~d} \mathbf{u}^{n}}\right|_{\Omega_{e}}=\mu_{0} \nabla \mathbf{H}_{0} \frac{\mathrm{d} G_{e}}{\mathrm{du}^{n}} .
$$

Computation of $\frac{\mathrm{dM}}{\mathrm{du}^{n}}$ : In the linear case, $\frac{\mathrm{d} \mathbf{M}}{\mathrm{d} \mathbf{u}^{n}}$ can be written as

$$
\frac{\mathrm{d} \mathbf{M}}{\mathrm{d} \mathbf{u}^{n}}=\chi \frac{\mathrm{d} \mathbf{H}}{\mathrm{du}^{n}}
$$

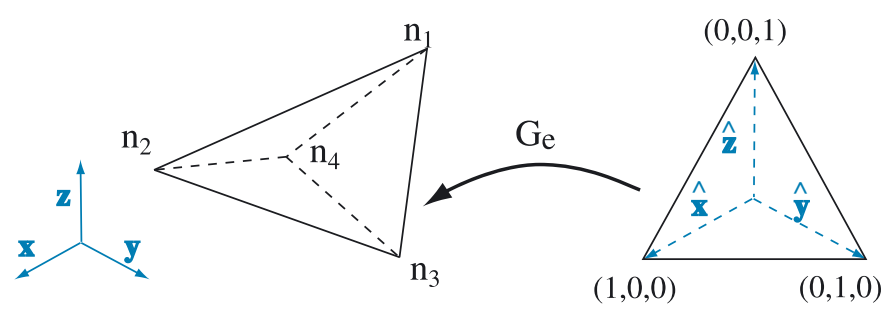

Figure 3. The change of basis between the physical and reference tetrahedral elements. 
With Equation (2) and the discretization (5) of the potential $\Phi$, we have

$$
\frac{\mathrm{d} \mathbf{H}}{\mathrm{d} \mathbf{u}^{n}}=-\sum_{i}\left(\frac{\mathrm{d} \Phi_{i}}{\mathrm{~d} \mathbf{u}^{n}} \nabla \alpha_{i}+\Phi_{i} \frac{\mathrm{d} \nabla \alpha_{i}}{\mathrm{du}^{n}}\right)
$$

where the derivatives of the gradients of the shape functions $\frac{\mathrm{d} \boldsymbol{\nabla} \alpha_{i}}{\mathrm{~d} \mathbf{u}^{n}}$ are obtained by relation (44). Finally, the $\frac{\mathrm{d} \Phi_{i}}{\mathrm{~d} \mathbf{u}^{n}}$ are computed by resolution of the system of algebraic equations:

$$
([I]+[A(\chi)]) \frac{\mathrm{d} \boldsymbol{\Phi}}{\mathrm{d} \mathbf{u}^{n}}=\frac{\mathrm{d} \boldsymbol{\Phi}_{0}}{\mathrm{~d} \mathbf{u}^{n}}-\left.\frac{\partial[A(\chi)]}{\partial \mathbf{u}^{n}}\right|_{\boldsymbol{\Phi}} \boldsymbol{\Phi} .
$$

$\frac{\mathrm{d} \boldsymbol{\Phi}_{0}}{\mathrm{~d} \mathbf{u}^{n}}$ is the vector of the derivative of the DOFs of the potential $\Phi_{0}$ with respect to the nodal displacement $\mathbf{u}^{n}$, and its computation is detailed in the next paragraph. The derivative of the interaction matrix $\left.\frac{\partial[A(\chi)]}{\partial \mathbf{u}^{n}}\right|_{\boldsymbol{\Phi}}$ must consider the mesh distortion. The analytical formula can not be used to compute the integral (36) when the computation point $\mathbf{r}$ is a node of the integration element [20]. In this case, a finite difference method can be used:

$$
\left.\frac{\partial\left[A\left(\chi, \mathbf{r}_{n}\right)\right]}{\partial \mathbf{u}^{n}}\right|_{\boldsymbol{\Phi}} \approx \frac{\left[A\left(\chi, \mathbf{r}_{n}+p \mathbf{e}_{u}\right)\right]-\left[A\left(\chi, \mathbf{r}_{n}-p \mathbf{e}_{u}\right)\right]}{2 p},
$$

where $p$ is the step of the method, and $\mathbf{e}_{u}$ is the direction of the virtual displacement $\mathbf{u}^{n}$ applied on the mesh node $n$ of coordinates $\mathbf{r}_{n}$.

Computation of $\frac{\mathbf{d} \Phi_{0}}{\mathbf{d u} u^{n}}$ : The vector $\frac{\mathrm{d} \boldsymbol{\Phi}_{0}}{\mathrm{~d} \mathbf{u}^{n}}$ is the solution of the derivation of the system of algebraic equations (11) with respect to the nodal displacement $\mathbf{u}^{n}$ :

$$
[D] \frac{\mathrm{d} \boldsymbol{\Phi}_{0}}{\mathrm{~d}^{n}}=\frac{\mathrm{d} \mathbf{C}}{\mathrm{du}^{n}}-\frac{\mathrm{d}[D]}{\mathrm{d}^{n}} \boldsymbol{\Phi},
$$

where

$$
\begin{gathered}
\left(\frac{\mathrm{d} \mathbf{C}}{\mathrm{d} \mathbf{u}^{n}}\right)_{i}=-\sum_{e} \int_{\hat{\Omega}}\left[\left(\frac{\mathrm{d} J_{e}^{-T}}{\mathrm{~d} \mathbf{u}^{n}} \hat{\nabla} \hat{\alpha}_{i} \cdot \mathbf{H}_{0}+\nabla \alpha_{i} \cdot \nabla \mathbf{H}_{0} \frac{\mathrm{d} G_{e}}{\mathrm{~d} \mathbf{u}^{n}}\right)\left|J_{e}\right|+\nabla \alpha_{i} \cdot \mathbf{H}_{0} \frac{\mathrm{d}\left|J_{e}\right|}{\mathrm{d} \mathbf{u}^{n}}\right] \mathrm{d} \Omega, \\
\left(\frac{\mathrm{d}[D]}{\mathrm{d} \mathbf{u}^{n}}\right)_{i j}=\sum_{e} \int_{\hat{\Omega}}\left[\left(\frac{\mathrm{d} J_{e}^{-T}}{\mathrm{~d} \mathbf{u}^{n}} \hat{\nabla} \hat{\alpha}_{i} \cdot \nabla \alpha_{j}+\nabla \alpha_{i} \cdot \frac{\mathrm{d} J_{e}^{-T}}{\mathrm{~d} \mathbf{u}^{n}} \hat{\nabla} \hat{\alpha}_{j}\right)\left|J_{e}\right|+\nabla \alpha_{i} \cdot \nabla \alpha_{j} \frac{\mathrm{d}\left|J_{e}\right|}{\mathrm{d} \mathbf{u}^{n}}\right] \mathrm{d} \Omega .
\end{gathered}
$$

Once the nodal magnetic forces (46) are computed, the magnetic force density $\mathbf{f}$ is obtained by a linear interpolation from its expression $\mathbf{f}^{n}$ at the mesh node $n$ :

$$
\mathbf{f}^{n}=\mathbf{F}^{n}\left(\sum_{e \in \mathcal{E}_{n}} \frac{V_{e}}{E_{e}}\right)^{-1},
$$

where $V_{e}$ and $E_{e}$ are respectively the volume and the number of nodes of the mesh element $e$, and $\mathcal{E}_{n}$ is the element set containing the node $n$.

To conclude this section, the proposed local application of the virtual work principle to compute the nodal magnetic force required the resolution of both systems of algebraic equations (53) and (51) for each mesh node and for each direction. Considering the linear case, the matrices on the left hand side of both systems (53) and (51) are respectively the same as those of both systems (11) and (6). In this case, the resolution processes could be kept modifying only the right hand member. 


\section{APPLICATIONS}

\subsection{Hollow sphere}

Let us consider a hollow ferromagnetic sphere with a linear magnetic behavior and placed in a magnetic field generated by a circular coil (Figure 4). The ferromagnetic domain is meshed using 9812 tetrahedral elements. The previous VIM (4) is used to solve this first application for which an analytical solution can be found [5]. The computed magnetostatic field distribution is presented in Figure 5. The virtual work principle (46) is applied to compute the nodal magnetic forces for three different meshes (1924, 4614, and 9812 elements), and the results are given in Figure 5. Considering the rotational symmetry with respect to the axis $\mathbf{z}$, the exploitation can be made on an arbitrary section resulting from a cut plane including the axis $\mathbf{z}$. The normal surface force densities computed on the outer surface of the sphere by relation (56) is compared with the analytical solution (Figure 6).

The results show a good concordance between the simulations and the analytical solution for the local magnetic forces, which validates the local application of the virtual work principle in the framework of the VIM and in the linear case. The average computation times for each magnetic nodal force are 1.9, 5.3, and 11.2 s (Dell desktop computer with Intel Core 2 Duo E8400 3GHz CPU (Dell Computer Round Rock, Texas, USA)) using respectively the meshes with 1924, 4614, and 9812 elements. The computation times are provided for information only, because the source code is not

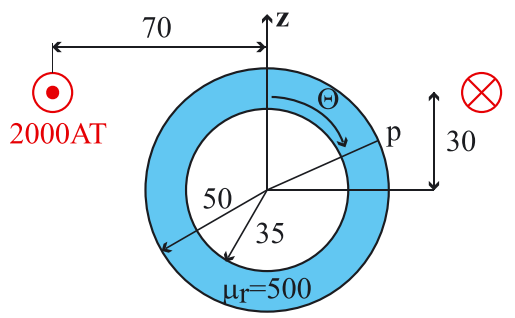

Figure 4. Description of the hollow sphere.
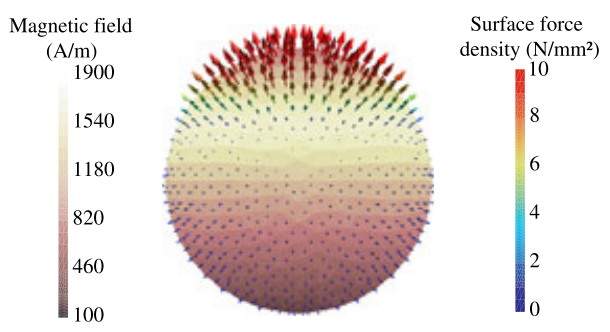

Figure 5. Computed magnetic field and force density.

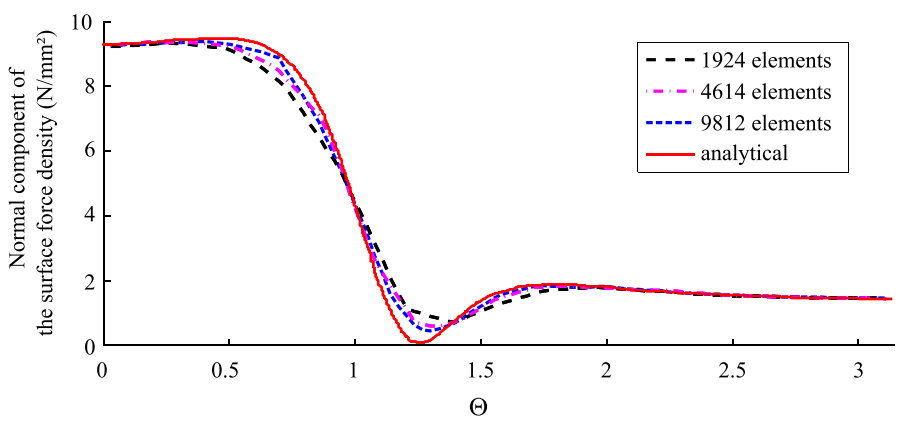

Figure 6. Results for the normal component of the computed surface magnetic force densities on the outer surface of the sphere. 
optimized. The computation of a magnetic nodal force is time consuming because of the assembly of the matrix $\left.\frac{\partial[A(\chi)]}{\partial \mathbf{u}^{n}}\right|_{\boldsymbol{\Phi}}$ on the right hand side of the system (51). The memory requirements are mainly dedicated to the storage of the interaction matrix $[A(\chi)]$ of the system (51).

\subsection{Contactor}

Let us consider the contactor described by the Figure 7.

Linear case: The magnetic behavior law of the ferromagnetic material is linear $(\chi=999)$, and the computation of local magnetic forces is investigated. The previous VIM (4) and the FEM with the software FLUX3D [21] are used to solve this problem. The virtual work principle (46) is applied to compute the nodal magnetic forces (Figure 8). The surface force density is computed along the segment $\mathrm{AB}$ by relation (56) and by the virtual work principle in the framework of the FEM for three different meshes (3914, 6253, and 18108 elements), and the results are given in the Figure 9.

For both methods, the results are inaccurate at the extremities of the segment AB and when the computation points pass in the air gap. The first effect is due to the geometric singularities. The causes of the second effect may be inaccuracies in the computation of the direction of the surface magnetic force density, especially its tangential component, which is negligible compared with its normal component. These inaccuracies are most important with the FEM because the discretization of the air gap is poor with the considered meshes. The VIM does not require the mesh of the free space; however, the range of these inaccuracies is larger because this method is based on interactions between all magnetized elements. Nevertheless, the results of both methods seem to converge to the same values and validate the proposed formulation. Its main drawback is heavy computation time due to great number of system resolutions (53) and (37).

Nonlinear case: The magnetic behavior law of the ferromagnetic material is nonlinear such as

$$
\|\mathbf{M}(\|\mathbf{H}\|)\|=\frac{2 J_{s}}{\mu_{0} \pi} \arctan \left(\frac{\pi\left(\mu_{r}-1\right) \mu_{0}\|\mathbf{H}\|}{2 J_{s}}\right)
$$
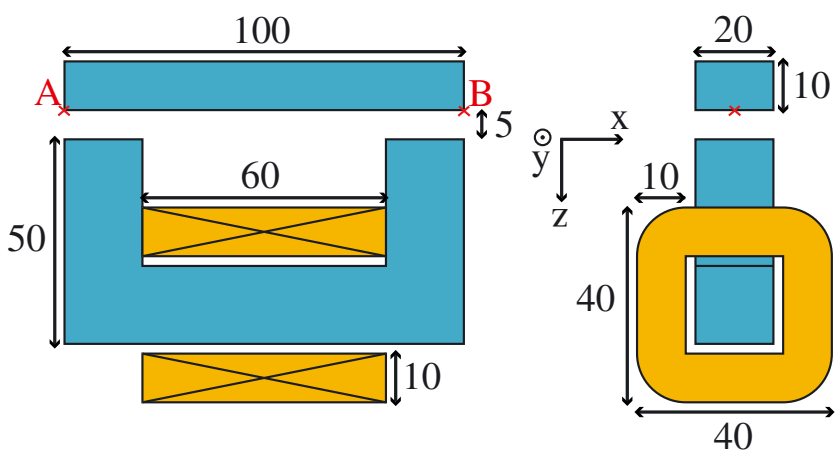

Figure 7. Description of the contactor.

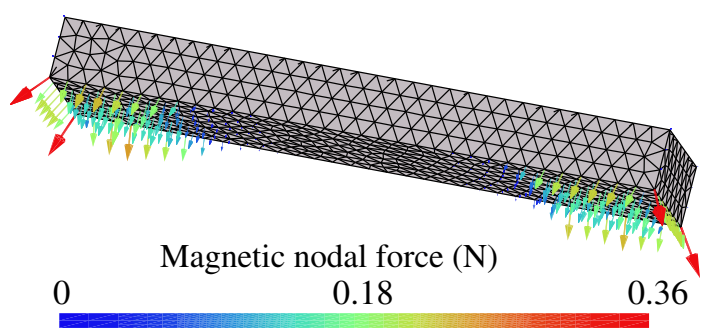

Figure 8. Nodal magnetic forces. 

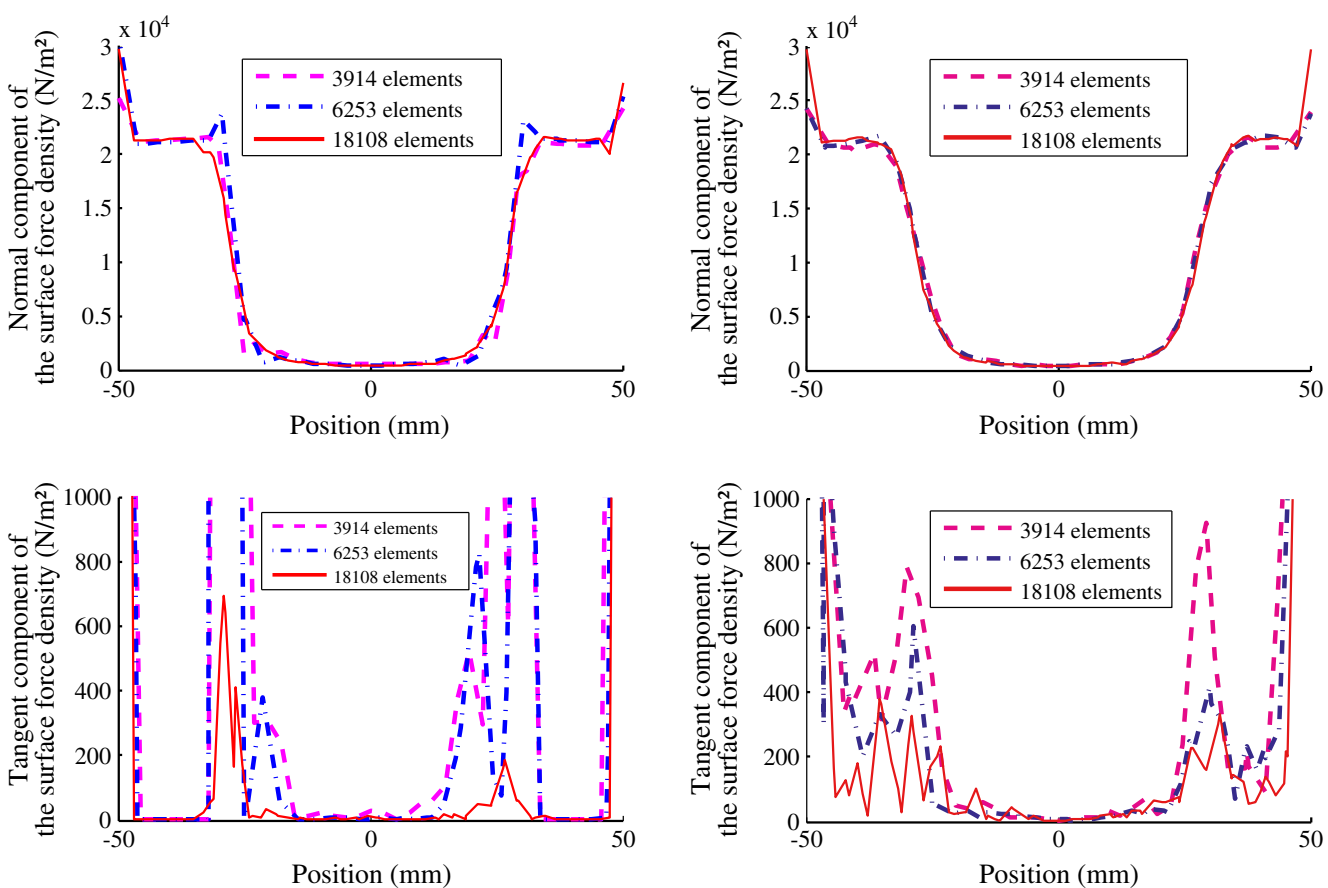

(a) Finite element method.

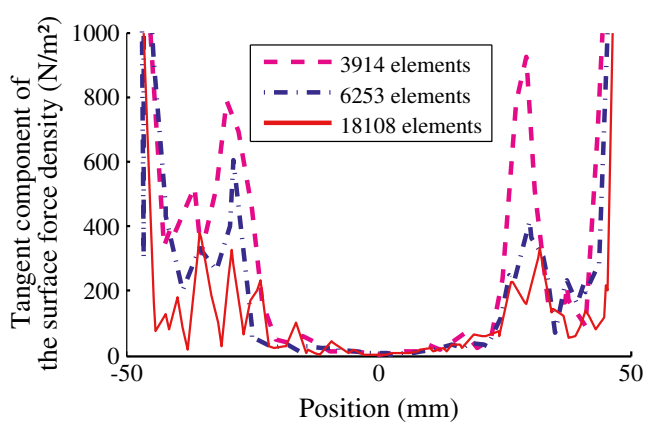

(b) Volume integral method

Figure 9. Results for the computed surface magnetic force density along the segment AB.

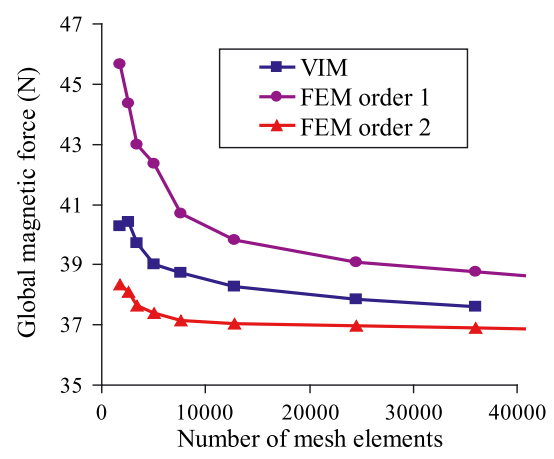

(a) Comparison with the finite element method.

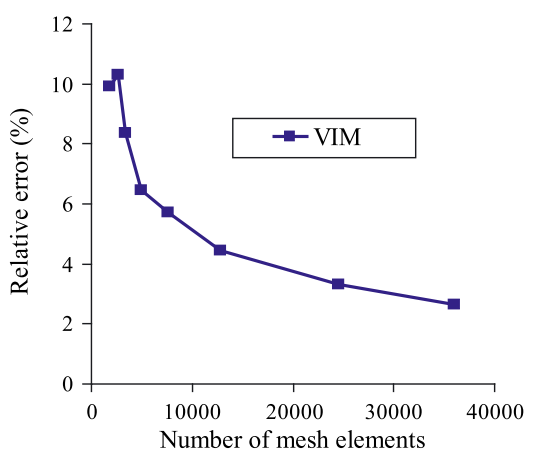

(b) Relative error with the reference value.

Figure 10. Results for the computed global magnetic force acting of the top piece of the contactor.

where $J_{s}=1.8 \mathrm{~T}$, and $\mu_{r}=2500$. The global magnetic force acting on the top piece of the contactor is investigated. The virtual work principle is then associated to a displacement of the entire top piece of the contactor. The FEM with first-order and second-order discretizations and the VIM are applied. Taking into account the symmetry of the problem, only the force component along the axis $\mathbf{z}$ is considered, the others being zero. The evolutions of the computed global magnetic force in function of the number of mesh elements in the contactor are compared in Figure 10. The reference value is obtained by the FEM [22] with a second-order discretization and a fine mesh $(185,000$ mesh elements in the contactor) and is $36.64 \mathrm{~N}$ along the axis $\mathbf{z}$.

The results show that the virtual work principle applied to the VIM provides computed global magnetic forces that converge relatively quickly toward a value. However, this convergence is much faster with a FEM and a second-order discretization. Moreover, the relative error between the reference value $36.64 \mathrm{~N}$ and the results provided by the VIM is less than $3 \%$ for a mesh with around 35, 000 elements. 
Table I. Computation times (personal computer with Intel Xeon E5-2690 2.9GHz CPU).

\begin{tabular}{lrrrrrrr}
\hline Number of mesh elements & 1795 & 2620 & 3436 & 4986 & 7566 & 12,785 & 24,508 \\
Resolution with NR method (s) & 71 & 103 & 157 & 390 & 708 & 2085 & 7266 \\
Global magnetic force (s) & 19 & 33 & 41 & 57 & 76 & 171 & 445 \\
\hline
\end{tabular}

NR, Newton-Raphson.

Nevertheless, the limitation of the full interaction matrix (8) does not allow to increase the number of mesh elements with the VIM; however, this represents a mesh with several hundreds of thousands of elements with FEM, which requires the air mesh. The computation times for the nonlinear resolution with the NR method and for the global magnetic force are given in Table I. The computation of the global magnetic force is time consuming because of the assembly of the matrix $\left.\frac{\partial[A(\chi)]}{\partial \mathbf{u}}\right|_{\boldsymbol{\Phi}}$ on the right hand side of the system (37) and the computation of the gradient of the source magnetic field $\nabla \mathbf{H}_{0}$ using a finite difference method at each Gauss point in order to numerically integrate the terms (48) and (54). The memory requirements are mainly dedicated to the storage of the matrices $\left[A\left(\frac{\mathrm{d} \mathbf{M}}{\mathrm{dH}}\right)\right]$ and $\left.\frac{\partial[A(\chi)]}{\partial \mathbf{u}}\right|_{\boldsymbol{\Phi}}$ of system (37).

\section{CONCLUSION}

The application of virtual work principle to compute the magnetic force densities has been adapted to the framework of the VIMs for which the air is not meshed. It is an attractive alternative to the FEM for devices that contain a huge volume of free space. The proposed formulation gives accurate results to compute local and global magnetic forces respectively in the linear and nonlinear cases. Its implementation could be improved especially to avoid the use of finite differences. The perspectives are to use compression techniques to avoid the storage limitation of the full interaction matrix and to extend the local magnetic force computation to the nonlinear case. The proposed approach could also be advantageously used in the framework of a magneto-mechanical coupling in which the same mesh is used to solve the magnetic and mechanical parts.

\section{REFERENCES}

1. Rakotoarison HL, Ardon V, Chadebec O, Delinchant B, Guerin S, Coulomb JL. Formal sensitivity computation of magnetic moment method. IEEE Trans Magn 2008; 44(6):1014-1017.

2. Trowbridge B. Integral equations in electromagnetics. Int $J$ Numer Model El 1996; 9(1-2):3-17.

3. Cugat O, Delamare J, Reyne G. Magnetic micro-actuators and systems (magmas). IEEE Trans Magn 2003; 39(5):3607-3612.

4. Kettunen L, Forsman K, Levine D, Gropp W. Volume integral equations in non-linear 3-d magnetostatics. Int J Numer Meth Eng 1995; 38(16):2655-2675.

5. Ren Z. Comparison of different force calculation methods in $3 \mathrm{~d}$ finite element modelling. IEEE Trans Magn 1994; 30(5): 3471-3474.
6. Henrotte F, Deliège G, Hameyer K. The eggshell approach for the computation of the electromagnetic forces in $2 \mathrm{~d}$ and 3d. COMPEL 2004; 23(4):996-1005.

7. Vandevelde L, Melkebeek JAA. A survey of magnetic force distributions based on different magnetization models and on the virtual work principle. IEEE Trans Magn 2001; 37(5): 3405-3409.

8. Bossavit A. Virtual power principle and maxwell's tensor: which comes first? COMPEL 2011; 30(6):1804-1814.

9. Ren Z, Razek A. Local force computation in deformable bodies using edge elements. IEEE Trans Magn 1992; 28(2):1212-1215.

10. Hantila F, Cranganu-Cretu B, Preda G, Miya K. Force evaluation formula for integral methods of magnetic field computation. Int
$J$ Appl Electrom 2002; 14(1-4): 3-8.

11. Kalimov A, Svedentsov M. Application of a hybrid integrodifferential method for analysis of thin magnetic shields. IEEE Trans Magn 1998; 34(5): 2453-2456.

12. Han L, Tong LS, Yang J. Integral equation method using total scalar potential for the simulation of linear or nonlinear 3D magnetostatic field with open boundary. IEEE Trans Magn 1994; 30(5): 2897-2900.

13. Carpentier A, Chadebec O, Galopin N, Meunier G, Bannwarth B. Resolution of nonlinear magnetostatic problems with a volume integral method using the magnetic scalar potential. IEEE Trans Magn 2013; 49(5):1685-1688.

14. Greengard L, Rokhlin V. A fast algorithm for particle simulations. 
J Comput Phys 1987; 73(2): 325-348.

15. Bebendorf $\mathrm{M}$, Rjasanow $\mathrm{S}$. Adaptive low-rank approximation of collocation matrices. Computing 2003; 70: $1-24$.

16. Rubeck C, Yonnet JP, Allag H, Delinchant B, Chadebec O. Analytical calculation of magnet systems: magnetic field created by charged triangles and polyhedra. IEEE Trans Magn 2013; 49(1): 144-147.
17. Urankar LK. Vector potential and magnetic field of current-carrying finite arc segment in analytical form, part i: filament approximation. IEEE Trans Magn 1980; 16(5):1283-1288.

18. Woodson HH, Melcher JR. Electromechanical Dynamics, Part II: Fields, Forces and Motion. John Wiley and Sons: New York, 1968.

19. Stratton JA. Electromagnetic Theory. Mcgraw Hill Book Company: New York, 1941.
20. Durand E. Electrostatique. Tome 1: Les Distributions. Masson: Paris, 1964.

21. Flux3D. CEDRAT, 2013. Available from: www.cedrat.com [Accessed date 2013].

22. Coulomb JL. A methodology for the determination of global electromechanical quantities from a finite element analysis and its application to the evaluation of magnetic forces, torques and stiffness. IEEE Trans Magn 1983; 19(6):2514-2519. 